\title{
DIAGNÓSTICOS DE ENFERMAGEM NO PERÍODO TRANSOPERATÓRIO: MAPEAMENTO CRUZADO
}

\author{
Nursing diagnoses in the perioperative period: cross mapping \\ Diagnóstico de enfermería en el periodo transoperatorio: mapeo cruzado
}

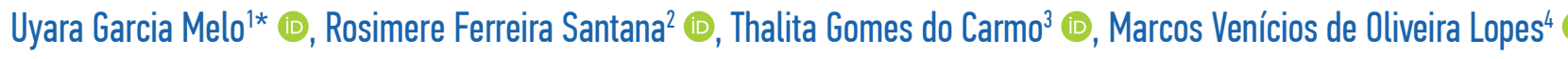

RESUMO: Objetivo: Mapear os diagnósticos de enfermagem da taxonomia North American Nursing Diagnosis Association International (NANDA-I) em pacientes no período transoperatório. Método: Estudo do tipo transversal, documental, dos registros de enfermagem no período transoperatório de um hospital da rede suplementar de saúde do município de Niterói, Rio de Janeiro, desenvolvido por meio da ferramenta metodológica mapeamento cruzado. Resultados: Foram avaliados 65 prontuários. A maior parte da amostra foi composta de mulheres com média de idade de 50,1 anos e submetidas a cirurgias eletivas. Quanto à especialidade cirúrgica, os procedimentos mais realizados foram gerais e urológicos. Os termos mais encontrados foram bisturi elétrico, placa de bisturi, anestesia geral e entubação. Pelo painel de especialistas, obtiveram-se como diagnósticos prevalentes: risco de infecção de sítio cirúrgico ( $84,6 \%)$, risco de integridade da pele prejudicada $(78,5 \%)$, integridade tissular prejudicada $(63,1 \%)$ e risco de aspiração $(58,5 \%)$. Conclusão: Os diagnósticos de enfermagem encontrados são prioritariamente de risco. Logo, carecem de identificação precoce e de intervenções para evitar danos e prevenir complicações, como o retardamento na recuperação cirúrgica. Palavras-chave: Diagnósticos de enfermagem. Enfermagem perioperatória. Terminologia padronizada em enfermagem.

ABSTRACT: Objective: To map nursing diagnoses of the North American Nursing Diagnosis Association International (NANDA-I) taxonomy in patients during the perioperative period. Method: This is a cross-sectional and documentary study on nursing records covering the perioperative period of a hospital part of the private healthcare system of Niterói, Rio de Janeiro, developed by the cross-mapping methodological tool. Results: 65 medical records were evaluated. Most of the sample consisted of women with a mean age of 50.1 years and who underwent elective surgeries. Regarding the surgical specialty, the most performed procedures were general and urological surgeries. The most found terms were electric scalpel, scalpel plate, general anesthesia, and intubation. From the group of specialists, the prevalent diagnoses obtained were: risk for surgical site infection (84.6\%), risk for impaired skin integrity (78.5\%), impaired tissue integrity (63.1\%), and risk for aspiration (58.5\%). Conclusion: Nursing diagnoses that are primarily of risk were observed. Therefore, they lack early identification and interventions to avoid damages and prevent complications such as delayed surgical recovery.

Keywords: Nursing diagnosis. Perioperative nursing. Standardized nursing terminology.

RESUMEN: Objetivo: Mapear los diagnósticos de enfermería de la taxonomía NANDA-I en pacientes en el período transoperatorio. Método: Estudio documental transversal de registros de enfermería en el período transoperatorio de un hospital de la Red de Salud Complementaria de la ciudad de Niterói, Río de Janeiro. Fue desarrollado utilizando la herramienta metodológica de mapeo cruzado. Resultados: se evaluaron 65 historias clínicas. La mayor parte de la muestra estaba compuesta por mujeres, con una edad media de 50,1 años y sometidas a cirugía electiva. En cuanto a la especialidad quirúrgica, los procedimientos más realizados fueron generales y urológicos. Los términos más comúnmente encontrados fueron bisturí eléctrico, placa de bisturí, anestesia general e intubación. El panel de expertos obtuvo los siguientes diagnósticos frecuentes: riesgo de infección del sitio quirúrgico (84,6\%), riesgo de integridad de la piel deteriorada (78,5\%), integridad del tejido deteriorada (63,1\%) y riesgo de aspiración (58.5\%). Conclusión: los diagnósticos de enfermería encontrados son principalmente de riesgo, por lo que necesitan identificación e intervenciones tempranas para prevenir daños y complicaciones, como la recuperación quirúrgica tardía.

Palabras clave: Diagnóstico de enfermería. Enfermería perioperatoria. Terminología normalizada de enfermería.

'Enfermeira. Residente em Centro Cirúrgico e Central de Esterilização de Materiais, Hospital Sírio-Libanês - São Paulo (SP), Brasil.

${ }^{2}$ Ṕs-doutora em Enfermagem. Professora associada do Departamento de Enfermagem Médico-Cirúrgico da Escola de Enfermagem Aurora de Afonso Costa da Universidade Federal Fluminense (UFF) - Niterói (RJ), Brasil ${ }^{3}$ Doutora em Enfermagem. Professora adjunta do Departamento de Enfermagem Médico-Cirúrgico da Escola de Enfermagem Aurora de Afonso Costa da UFF - Niterói (RJ), Brasil.

«Pós-doutor em Enfermagem. Professor associado da Universidade Federal do Ceará (UFC) - Fortaleza (CE), Brasil.

*Autora correspondente: uyara.gmelo@gmail.com

Recebido: 25/02/2019 - Aprovado: 04/10/2019

DOI: $10.5327 / Z 1414-4425201900040004$ 


\section{INTRODUÇÃO}

O Manual de Cirurgia Segura ${ }^{1}$ destaca, com base em dados de 56 países, que o volume anual de cirurgias de grande porte foi estimado entre 187 e 281 milhões, o que representa aproximadamente uma cirurgia para cada 25 pessoas por ano. Esse é um volume amplo, que vem crescendo nas últimas décadas, em razão da alta incidência de agravos traumáticos, do envelhecimento populacional e do consequente aumento da prevalência de doenças crônicas, fatores que implicam a necessidade crescente de intervenções cirúrgicas ${ }^{2}$. Entretanto, esses avanços também elevaram, de modo significativo, a ocorrência de erros que podem resultar em danos para os pacientes e, por conseguinte, levar a implicações significativas na saúde pública.

Diante disso, faz-se preciso a sistemática implementação do processo de enfermagem, a fim de identificar precocemente os problemas já existentes e os potenciais, de maneira a intervir para reduzir danos e prejuízos. Logo, o Conselho Federal de Enfermagem (Cofen) tem legislado sobre as competências dos profissionais de enfermagem e sobre as anotações que devem ser documentadas. Destaca-se a Resolução $n^{\circ} 358 / 2009$, que considera a sistematização da assistência de enfermagem (SAE) como método do processo de trabalho que evidencia a contribuição do enfermeiro na atenção à saúde da população, aumentando sua visibilidade e seu reconhecimento profissional ${ }^{3}$.

A SAE visa orientar as atividades da equipe de enfermagem e é responsável por organizar o trabalho da equipe quanto ao método a ser aplicado, ao pessoal e aos instrumentos necessários para que seja possível a operacionalização do processo de enfermagem (PE). Até a década de 1960, a enfermagem no Centro Cirúrgico (CC) consistia, predominantemente, em instrumentalização, atendimento das solicitações da equipe médica e ações administrativas relacionadas ao bom desenvolvimento do ato anestésico-cirúrgico. Porém, em 1990, foi proposta por Castellanos e Jouclas a aplicação do PE no cuidado ao paciente cirúrgico, com o propósito de promover assistência integral, continuada, participativa, individualizada e documentada. Nesse modelo de assistência, cada paciente é visto de modo singular, e a intervenção de enfermagem objetiva promover a continuidade do cuidado, além de proporcionar a participação da família do paciente e possibilitar a avaliação da assistência prestada. Suas idealizadoras denominaram esse processo de sistematização de assistência de enfermagem perioperatória (SAEP)².
Nesse momento, torna-se imperioso diferenciar SAE de PE: a primeira existe somente no contexto brasileiro, e o segundo é reconhecido internacionalmente como o modo operante do processo de trabalho do enfermeiro. O PE é uma atividade privativa do enfermeiro, que inclui "coleta de dados, diagnóstico de enfermagem, planejamento, implementação e avaliação"”.

Um diagnóstico de enfermagem proporciona a base para a seleção das intervenções de enfermagem, de forma a atingir resultados pelos quais o enfermeiro é responsável ${ }^{4}$. A North American Nursing Diagnosis Association International (NANDA-I) formalizou um sistema de classificação que visa descrever e desenvolver uma fundamentação científica que ofereça o alicerce para que a equipe de enfermagem selecione as intervenções adequadas para cada paciente ${ }^{5}$. Portanto, a NANDA-I sugere uma maneira de classificar e categorizar áreas de preocupação para a enfermagem, apresentando 244 diagnósticos, agrupados em 13 domínios, os quais se subdividem em 47 classes ${ }^{4}$.

Contudo, ainda existem poucos estudos sobre os diagnósticos de enfermagem válidos para o período transoperatório na literatura, e, assim, o seu uso na prática clínica ainda tem sido tímido.

Desse modo, para denominar os benefícios da implementação do PE, torna-se essencial estabelecer os termos de enfermagem tomando como base os registros já existentes nos prontuários dos pacientes, para que, a partir daí, se possa compará-los com as taxonomias de enfermagem, a fim de identificar a prevalência dos diagnósticos no cotidiano da enfermagem. Padronizar a nomenclatura da prática de enfermagem pode favorecer a comunicação entre os membros da equipe e de saúde, promover a continuidade da assistência e fomentar a construção de uma enfermagem fundamentada em princípios científicos, com raciocínio clínico.

\section{OBJETIVO}

Mapear os diagnósticos de enfermagem da taxonomia NANDA-I em pacientes no período transoperatório.

\section{MÉTODO}

Trata-se de um estudo transversal, documental, dos registros de enfermagem contidos nos prontuários de pacientes no período transoperatório em um hospital da rede suplementar 
de saúde do município de Niterói, Rio de Janeiro. O estudo foi desenvolvido por meio da ferramenta metodológica mapeamento cruzado (cross mapping), que tem por finalidade realizar uma comparação entre os termos utilizados no cotidiano do serviço e o sistema de classificação de diagnósticos de enfermagem NANDA-I.

Selecionou-se como local de estudo um hospital geral de grande porte da rede suplementar de saúde da cidade de Niterói. A escolha de um hospital dessa rede deu-se em razão de este possuir um enfermeiro dentro da sala de operação, o qual registra e evolui o paciente durante o período transoperatório.

A coleta de dados foi realizada por meio de um instrumento desenvolvido pelos pesquisadores, a fim de padronizar os dados a serem coletados nos registros de enfermagem, nos quais as informações do paciente e do período transoperatório foram descritas conforme a especificidade de cada paciente.

Os prontuários de pacientes no período transoperatório foram selecionados por amostragem intencional, consecutiva, sendo coletados os registros de todos os pacientes disponíveis para coleta de dados nos dias de disponibilidade do pesquisador. O período de coleta de dados foi de agosto a setembro de 2018, e o pesquisador compareceu à unidade 12 vezes, em dias alternados, permanecendo ali entre quatro e cinco horas por dia.

Critérios de inclusão: pacientes em atendimento no intraoperatório no momento da coleta e maiores de 18 anos. Critérios de exclusão: prontuários que, por ocasião de rotinas internas do hospital, estivessem indisponíveis; e prontuários que não contivessem registro de enfermagem do período transoperatório, completos e/ou legíveis.

No mês de setembro, o hospital sede do estudo realizou 758 cirurgias, sendo a amostra limitada a 65 pacientes.
Para coleta e análise dos dados, foi elaborado um roteiro contendo:

- dados de caracterização do paciente e descrição dos termos livres encontrados nos registros de enfermagem durante o período transoperatório;

- comparação entre os termos e a classificação NANDA-I;

- envio dos formulários para análise individual pelos peritos para traçar os diagnósticos de acordo com os termos livres;

- painel de especialistas para consenso dos diagnósticos, de acordo com os termos livres.

A utilização do cross mapping respeitou a apresentação dos termos a serem comparados e as adaptações necessárias levando em conta as regras consideradas neste estudo ${ }^{6}$ e apresentadas no Quadro 1.

Após a avaliação dos registros, elaboraram-se diagnósticos de enfermagem com base nos termos destacados pelo pesquisador, os quais foram registrados em formulários e, em seguida, lançados em uma planilha do Excel. Os fatores relacionados e de risco foram identificados por meio da interpretação de termos associados, sinônimos ou conceitos similares. Para tanto, realizou-se a análise de combinação, adaptada de forma que, se o termo encontrado combinasse com o termo do sistema de classificação, a combinação era designada como exata. Entretanto, se os termos fossem sinônimos, conceitos similares ou relacionados, a combinação era designada como parcial. Os termos que não apresentavam semelhança com o sistema de classificação nem possuíam combinação foram coletados dos registros, porém a maioria não foi utilizada.

Quadro 1. Regras de utilização do cross mapping.

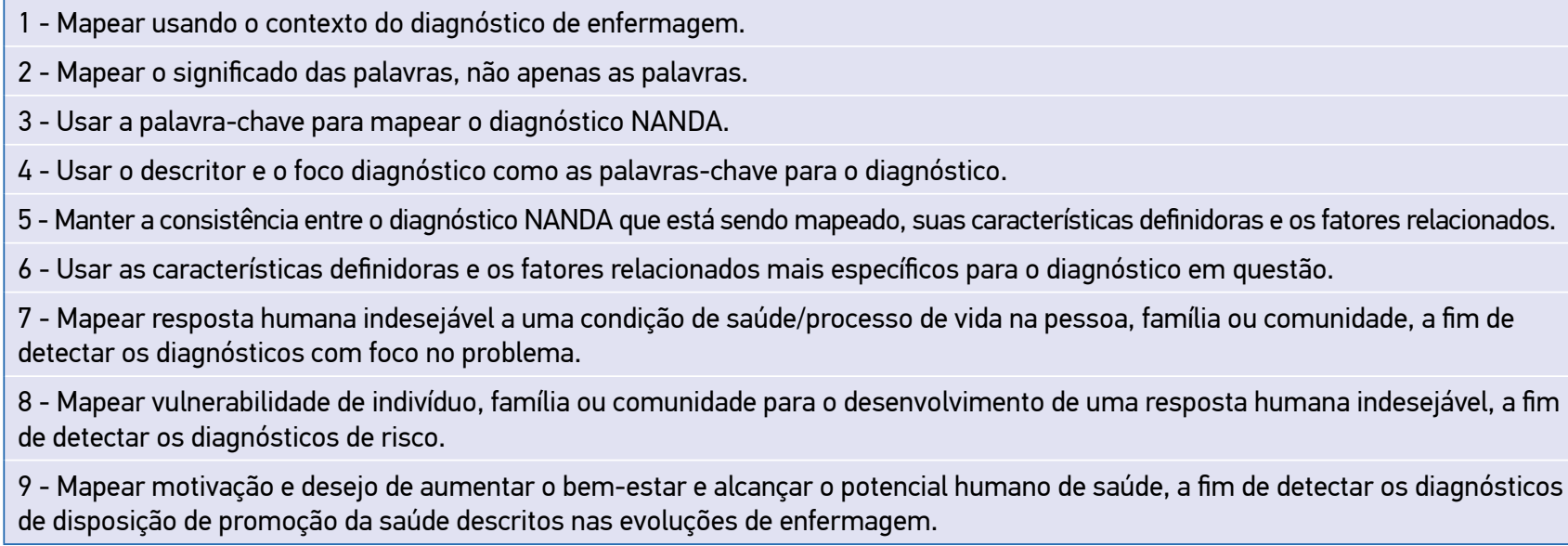


Após a finalização da coleta de dados, os formulários foram encaminhados para peritos, para que estes traçassem diagnósticos de enfermagem segundo a classificação NANDA-I. Assim, a terceira etapa correspondeu à análise feita por três peritos: o próprio pesquisador, um especialista em diagnóstico de enfermagem e um especialista em enfermagem de CC. Os diagnósticos foram traçados por intermédio da relação entre os termos levantados nos prontuários dos pacientes incluídos na amostra e os termos encontrados nos fatores relacionados e nas características definidoras dos diagnósticos contidos na classificação NANDA-I.

Ainda, optou-se pela realização de uma quarta etapa, que consistiu em um painel de especialistas para consenso dos diagnósticos de enfermagem identificados individualmente com base nos termos livres, momento no qual os peritos apresentaram concordância ou não sobre cada diagnóstico para cada paciente. Nessa etapa, se um diagnóstico era levantado por apenas um perito, ele era classificado como não concordância; no caso de haver consenso entre dois ou os três peritos, classificava-se o diagnóstico como concordante. Essa etapa ocorreu em grupo e durou em torno de oito horas.

Por fim, os dados do painel de especialistas e a avaliação individual de cada perito foram lançados em um banco de dados e enviados para análise estatística.

As etapas da coleta e da análise dos dados estão representadas na Figura 1.

Cabe ressaltar que o presente projeto foi submetido à avaliação do Comitê de Ética do Hospital Universitário Antônio Pedro, instituição promotora, a qual está vinculada à Escola de Enfermagem Aurora de Afonso Costa, tendo sido aprovado em 11 de junho de 2018, via Plataforma Brasil, sob Certificado de Apresentação para Apreciação Ética (CAAE) 89858218.2.0000.5243 e protocolo número 2.705.126.

\section{RESULTADOS}

Na Tabela 1, apresentam-se dados referentes à caracterização do perfil dos participantes do estudo $(n=65)$ e das especialidades cirúrgicas às quais foram submetidos.

A maioria dos participantes era do sexo feminino (37/56,9\%), com média de idade de 50,1 anos; o participante mais jovem tinha 19 anos, e o mais idoso, 85. Quanto às cirurgias, a maioria foi eletiva (63/96,9\%), e as especialidades mais frequentes foram geral $(26 / 40,0 \%)$ e urologia $(17 / 26,2 \%)$. As cirurgias classificadas como geral são aquelas que compreendem as cirurgias da região abdominal (esôfago, estômago, intestinos, fígado, cólon, pâncreas, vesícula biliar e dutos biliares), cirurgias videolaparoscópicas e cirurgias do trauma.

A Tabela 2 apresenta os termos livres encontrados pelo pesquisador nos registros de enfermagem dos pacientes no período transoperatório.

Observa-se que os termos mais comuns foram bisturi elétrico e placa de bisturi - ambos aparecem na mesma

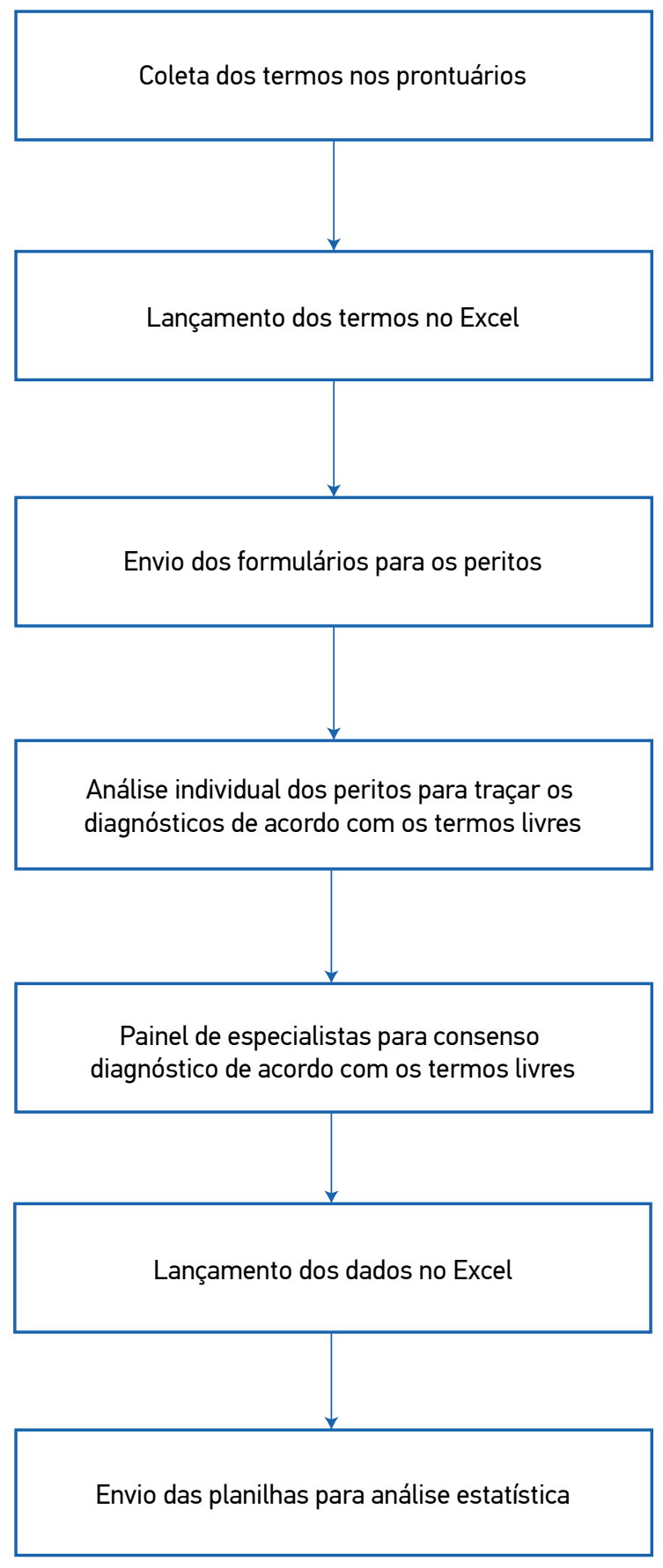

Figura 1. Etapas de coleta e análise dos dados. 
proporção (51/78,5\%). Além deles, foram encontrados, frequentemente, os termos anestesia geral $(40 / 61,5 \%)$ e entubação $(36 / 55,4 \%)$.

A Tabela 3 demonstra os diagnósticos de enfermagem segundo a concordância consensual realizada pelos peritos e pelo pesquisador.

A Tabela 3 mostra que, de acordo com o consenso realizado entre os peritos e o pesquisador, os diagnósticos de enfermagem prevalentes foram: risco de infecção de sítio cirúrgico, presente em 55 (84,6\%) pacientes do estudo; risco de integridade da pele prejudicada $(51 / 78,5 \%)$; integridade tissular prejudicada $(41 / 63,1 \%)$; e risco de aspiração $(38 / 58,5 \%)$.

\section{DISCUSSÃO}

Com os dados coletados, foi possível demonstrar os diagnósticos de enfermagem mais prevalentes no período transoperatório, o que auxilia a defesa de que, se identificados e tratados com acurácia, podem melhorar a qualidade do cuidado e diminuir os riscos e a ocorrência de eventos adversos ao paciente no intraoperatório.

Tabela 1. Pacientes que compuseram a amostra, segundo sexo biológico, classificação da cirurgia e especialidade cirúrgica.

\begin{tabular}{|c|c|c|c|}
\hline \multicolumn{2}{|l|}{ Variáveis } & \multicolumn{2}{|c|}{ n (\%) } \\
\hline \multicolumn{4}{|l|}{ Sexo biológico } \\
\hline \multicolumn{2}{|l|}{ Feminino } & \multicolumn{2}{|c|}{$37(56,9)$} \\
\hline \multicolumn{2}{|l|}{ Masculino } & \multicolumn{2}{|c|}{$28(43,1)$} \\
\hline \multicolumn{4}{|c|}{ Classificação da cirurgia } \\
\hline \multicolumn{2}{|l|}{ Eletiva } & \multicolumn{2}{|c|}{$63(96,9)$} \\
\hline \multicolumn{2}{|l|}{ Emergência } & \multicolumn{2}{|c|}{$2(3,1)$} \\
\hline \multicolumn{4}{|c|}{ Especialidade cirúrgica } \\
\hline \multicolumn{2}{|l|}{ Geral } & \multicolumn{2}{|c|}{$26(40,0)$} \\
\hline \multicolumn{2}{|l|}{ Urologia } & \multicolumn{2}{|c|}{$17(26,2)$} \\
\hline \multicolumn{2}{|l|}{ Neurologia } & \multicolumn{2}{|c|}{$8(12,3)$} \\
\hline \multicolumn{2}{|l|}{ Ortopedia } & \multicolumn{2}{|c|}{$5(7,7)$} \\
\hline \multicolumn{2}{|l|}{ Plástica } & \multicolumn{2}{|c|}{$4(6,2)$} \\
\hline \multicolumn{2}{|l|}{ Ginecologia } & \multicolumn{2}{|c|}{$3(4,6)$} \\
\hline \multicolumn{2}{|l|}{ Torácica } & \multicolumn{2}{|c|}{$1(1,5)$} \\
\hline \multicolumn{2}{|l|}{ Vascular } & \multicolumn{2}{|c|}{$1(1,5)$} \\
\hline Variáveis & Média & DP & $\mathbf{p}$ \\
\hline Idade & 50,1 & 17,9 & $<0,001$ \\
\hline
\end{tabular}

DP: desvio padrão.
Tabela 2. Distribuição dos termos livres encontrados nos prontuários dos participantes.

\begin{tabular}{|c|c|}
\hline Termos livres & n (\%) \\
\hline Bisturi elétrico & $51(78,5)$ \\
\hline Placa de bisturi & $51(78,5)$ \\
\hline Anestesia geral & $40(61,5)$ \\
\hline Entubação & $36(55,4)$ \\
\hline Sonda Foley & $22(33,8)$ \\
\hline $\begin{array}{l}\text { Proteção de proeminências } \\
\text { ósseas e áreas de apoio }\end{array}$ & $21(32,2)$ \\
\hline ELPO: alto risco & $21(33,3)$ \\
\hline Alergia & $19(29,2)$ \\
\hline Hipertensão/hipotensão & $19(29,2)$ \\
\hline Antibioticoprofilaxia não conforme & $18(27,7)$ \\
\hline $\begin{array}{l}\text { Protocolo de tromboembolismo } \\
\text { venoso (TEV): risco alto }\end{array}$ & $13(20,0)$ \\
\hline Tricotomia & $9(13,8)$ \\
\hline Reserva de sangue & $8(12,3)$ \\
\hline Protocolo TEV: risco moderado & $7(10,8)$ \\
\hline American Society of Anesthesiologists (ASA) III & $5(7,7)$ \\
\hline Uso de manguito da ortopedia & $4(6,2)$ \\
\hline Taquicardia/Bradicardia & $2(3,1)$ \\
\hline Hipotermia & $2(3,1)$ \\
\hline ASA IV & $1(1,5)$ \\
\hline ASA V & $1(1,5)$ \\
\hline
\end{tabular}

ELPO: escala de avaliação de risco para o desenvolvimento de lesões decorrentes do posicionamento cirúrgico do paciente.

Tabela 3. Diagnósticos com concordância consensual entre os especialistas e o pesquisador.

\begin{tabular}{|l|c|}
\hline Diagnóstico & $\mathbf{n}(\%)$ \\
\hline Risco de infecção de sítio cirúrgico & $55(84,6)$ \\
\hline Risco de integridade da pele prejudicada & $51(78,5)$ \\
\hline Integridade tissular prejudicada & $41(63,1)$ \\
\hline Risco de aspiração & $38(58,5)$ \\
\hline $\begin{array}{l}\text { Risco de lesão por posicionamento } \\
\text { perioperatório }\end{array}$ & $24(36,9)$ \\
\hline Risco de tromboembolismo venoso & $22(33,8)$ \\
\hline Risco de débito cardíaco diminuído & $19(29,2)$ \\
\hline Risco de resposta alérgica & $16(24,6)$ \\
\hline Eliminação urinária prejudicada & $16(24,6)$ \\
\hline Risco de recuperação cirúrgica retardada & $15(23,1)$ \\
\hline Risco de sangramento & $8(12,3)$ \\
\hline Risco de perfusão tissular periférica ineficaz & $5(7,7)$ \\
\hline Risco de lesão de trato urinário & $4(6,2)$ \\
\hline Risco de hipotermia perioperatória & $1(1,5)$ \\
\hline Padrão respiratório ineficaz & $1(1,5)$ \\
\hline
\end{tabular}


Com base no consenso entre peritos e pesquisador, verificou-se que os diagnósticos mais prevalentes foram: risco de infecção de sítio cirúrgico, risco de integridade da pele prejudicada, integridade tissular prejudicada e risco de aspiração. Nota-se que a maioria dos diagnósticos encontrados no período transoperatório foi de risco, ou seja, aqueles que apresentam julgamento clínico a respeito da suscetibilidade de um indivíduo desenvolver uma resposta indesejável a uma condição de saúde ${ }^{4}$.

Outra pesquisa também teve como resultado de diagnósticos no período transoperatório de cirurgias cardíacas maior número de diagnósticos de risco ${ }^{7}$. Sabe-se que um diagnóstico de risco não apresenta as mesmas consequências do diagnóstico real por não possuir características definidoras, entretanto seus fatores de risco, quando não tratados nem/ou prevenidos, podem culminar no diagnóstico em si. Daí a importância de uma assistência de enfermagem que busque associar as individualidades apresentadas pelos pacientes com os diagnósticos de enfermagem, de modo a implementar ações em busca de resultados que minimizem o tempo de internação e de recuperação do paciente ${ }^{8}$.

Outros autores ${ }^{9}$, durante o acompanhamento dos pacientes no período transoperatório, identificaram o diagnóstico de enfermagem de risco de lesão por posicionamento perioperatório em $100 \%$ da amostra. Em outro artigo $^{10}$, os mesmos autores do estudo anterior trataram da atenção ao paciente no período transoperatório, buscando identificar o diagnóstico de enfermagem mais frequente nesse período e descrever seus fatores de risco. A amostra contou com dez pacientes, de ambos os sexos, que foram submetidos a cirurgias gerais. O diagnóstico mais frequente foi o de risco de infecção, o qual ocorreu em $100 \%$ dos pacientes analisados ${ }^{10}$.

A escala de avaliação de risco para o desenvolvimento de lesões decorrentes do posicionamento cirúrgico do paciente (ELPO), criada por uma enfermeira brasileira, contém sete itens, com cinco subitens cada um, cuja pontuação varia de 1 a 5 pontos, apresentando pontuação total de 7 a 35 pontos, na qual, quanto maior o escore em que o paciente é classificado, maior o risco de desenvolver lesões por conta do posicionamento cirúrgico. A autora da ELPO ${ }^{11}$ identificou a associação entre a escala e o desenvolvimento de lesão perioperatória e evidenciou que, a cada ponto a mais em que o sujeito é classificado na escala, aumenta $44 \%$ a probabilidade de desenvolver lesão.
Além disso, a posição supina é mais anatômica, e as complicações relacionadas a essa posição ocorrem por conta do posicionamento inadequado e do tempo prolongado da cirurgia ${ }^{12}$. Já na posição litotômica, o paciente permanece em decúbito dorsal, com os membros inferiores abduzidos, apoiados sobre um suporte de pernas e elevados, formando um ângulo de aproximadamente $90^{\circ} \mathrm{com}$ a articulação do quadril. Essa posição oferece maior risco de complicações, em razão da pressão nas regiões sacra e lombar. Pacientes em posição litotômica apresentam maior risco de lesão $(59,72 \%)$ quando comparados aos pacientes colocados nas demais posições cirúrgicas ${ }^{2,11,12}$.

Em relação ao risco de infecção, um estudo ${ }^{13}$ mostra que ser classificado como American Society of Anesthesiologists (ASA) II, III e IV/V aumenta em 52, 134 e $89 \%$, respectivamente, as chances de desenvolver infecção de sítio cirúrgico (ISC) em comparação aos pacientes ASA I. Por outro lado, um estudo realizado em um hospital público de Belém (PA $)^{14}$ mostrou que a incidência de ISC aumentou à medida que o índice ASA também aumentou. A taxa de ISC em pacientes ASA I foi de 6,3\%; nos ASA II, de 10,5\%; e nos ASA III, de $100 \%(\mathrm{p} \leq 0,0001)$, o que torna o índice ASA significativamente capaz de influenciar a ISC. Daí a importância de se associar o dado coletado no instrumento com um diagnóstico de enfermagem e, consequentemente, com uma intervenção de enfermagem, para sistematicamente buscar atingir resultados. Essa é a verdadeira função de se identificar dados e registrá-los.

Em todos os artigos citados, os diagnósticos NANDA-I identificados pelos autores foram substituídos por seus referentes na versão atual da taxonomia (2018-2020), visto que os estudos se basearam em versões antigas da classificação NANDA-I.

Pode-se observar que a maioria dos diagnósticos de enfermagem encontrados no período transoperatório é de risco, ou seja, aqueles que podem ser evitados por meio da identificação precoce e da implementação de intervenções realizadas no plano de cuidados. Assim, a assistência de enfermagem deve estar voltada para o planejamento dos cuidados, considerando as evidências diagnósticas apresentadas pelos indivíduos. Dessa forma, ressalta-se a importância de a equipe de enfermagem utilizar as classificações taxonômicas no momento de seus registros, de modo a melhorar a assistência prestada ao paciente cirúrgico. 
Por fim, cabe declarar as limitações do estudo. Em primeiro lugar, o número reduzido de pesquisas que tratam dos diagnósticos de enfermagem no período perioperatório, permitindo maior discussão. Essa limitação era esperada, diante da especificidade do objeto da pesquisa e da escassez de publicações sobre a temática. Em segundo lugar, tem-se a baixa evidência dos estudos com especialistas, como os estudos de consenso, uma vez que, para a base da pirâmide de análise de qualidade de estudo, esse tipo de pesquisa é essencial para objetos da prática. Apesar disso, medidas de análise estatísticas e etapas individuais foram realizadas para diminuir o viés de detecção. Ademais, cuidados foram tomados para minimizar o viés de seleção, e coletaram-se os dados em dias e horários alternados. Embora não apresente cálculo amostral, por conta do tipo de estudo realizado, uma amostra maior tornaria a avaliação pelo perito dispendiosa e propensa ao erro, e as associações apresentadas demonstram que a amostra atendeu aos objetivos propostos.

\section{CONCLUSÃO}

Existem termos livres dos registros da prática cotidiana de enfermagem que remetem aos diagnósticos de enfermagem propostos pela taxonomia NANDA-I que são significantes para organizar e direcionar a qualidade do cuidado de enfermagem com os pacientes em período transoperatório.

Evidencia-se, portanto, que o enfermeiro, ao realizar a avaliação dos pacientes cirúrgicos por meio do $\mathrm{PE}$, deve julgar clínica e individualmente as necessidades e os fatores de risco apresentados pelos pacientes. Como pontuado nos resultados e na discussão deste estudo, verificou-se que a maioria dos diagnósticos encontrados no período transoperatório era de risco: risco de infecção de sítio cirúrgico, risco de integridade da pele prejudicada, integridade tissular prejudicada, risco de aspiração e risco de lesão por posicionamento perioperatório. Dessa forma, devem ser implementadas medidas de detecção precoce e intervenções preventivas de danos.

\section{REFERÊNCIAS}

1. Organização Mundial da Saúde. Segundo desafio global para a segurança do paciente: cirurgias seguras salvam vidas. Orientações para cirurgia segura da OMS. Rio de Janeiro: OMS; 2009.

2. Associação Brasileira de Enfermeiros de Centro Cirúrgico, Recuperação Anestésica e Centro de Material e Esterilização. Diretrizes de práticas em enfermagem cirúrgica e processamento de produtos para a saúde. 7ª ed. São Paulo: SOBECC; 2017.

3. Conselho Federal de Enfermagem. Resolução n 358, de 15 de outubro de 2009. Dispõe sobre a Sistematização da Assistência de Enfermagem e a implementação do Processo de Enfermagem [Internet]. Brasília; 2009 [acessado em 9 ago. 2017]. Disponível em: http://www.cofen.gov.br/resoluocofen-3582009_4384.html

4. Herdman H, Kamitsuru S. Diagnósticos de enfermagem da NANDA: definições e classificação 2018-2020. Porto Alegre: Artmed; 2018.

5. Carpenito LJ. Diagnósticos de enfermagem: aplicação à prática clínica. 8a ed. Porto Alegre: Artmed; 2003.

6. Lucena AF, Barros AL. Mapeamento cruzado: uma alternativa para análise de dados em enfermagem. Acta Paul Enferm. 2005;18(1):825. http://dx.doi.org/10.1590/S0103-21002005000100011

7. Galdeano LE, Rossi LA, Santos CB, Dantas RAS. Nursing diagnosis in the perioperative of cardiac surgery. Rev Esc Enferm USP. 2006;40(1):26-33. https://doi.org/10.1590/ S0080-62342006000100004
8. RemboldSM, Santana RF, Souza PA, SchwartzSMO. Nursing diagnosis Risk for Delayed Surgical Recovery (00246): concept clarification and definition of empirical referentes. Int J Nurs Know [Internet]. 2018 [acessado em 26 jul. 2019];29(4):263-8. Disponível em: https://onlinelibrary.wiley.com/doi/ abs/10.1111/2047-3095.12176. https://doi.org/10.1111/2047-3095.12176

9. Matos FGOA, Piccoli M. Diagnóstico de enfermagem: risco para lesão perioperatória por posicionamento identificado no período transoperatório. Ciênc Cuid Saúde [Internet]. 2004 [acessado 15 ago. 2017];3(2):195-201. Disponível em: https://doi.org/10.4025/cienccuidsaude.v3i2.5426

10. Piccoli M, Matos FGO. Nursing diagnosis: infection risk during the transsurgical period based on Levine's reference. RevSOBECC. 2004;9(3):25-30.

11. LopesCMM, Haas VJ, DantasRAS, OliveiraCG, Galvão CM. Escaladeavaliação de risco para lesões decorrentes do posicionamento cirúrgico. Rev Latino-Am Enfermagem. 2016;24:e2704. http://dx.doi.org/10.1590/1518-8345.0644.2704

12. Trevilato DD, Melo TC, Fagundes MABG, Caregnato RCA. Posicionamento cirúrgico: prevalência de risco de lesões em pacientes cirúrgicos. RevSOBECC. 2018;23(3):124-9. https://doi.org/10.5327/Z1414-4425201800030003

13. Carvalho RLR, Campos CC, Franco LMC, Rocha AM, Ercole FF. Incidência e fatores de risco para infecção de sítio cirúrgico em cirurgias gerais. Rev Latino-Am Enfermagem. 2017;25:1-8. http:// dx.doi.org/10.1590/1518-8345.1502.2848

14. Rodrigues ALDS, Miranda AC, Dourado CJC, Almeida DPR, Brito NB, Araújo RS. Avaliação de pacientes quanto à infecção de sítio cirúrgico, em um hospital público de Belém-PA. Rev Paraense Med [Internet]. 2014 [acessado em 26 jul. 2019];28(1):23-30. Disponível em: http://files.bvs.br/upload/S/0101-5907/2014/v28n1/a4160.pdf 